\title{
Co-encapsulation of a Drug with a Protein in Erythrocytes for Improved Drug Loading and Release: Phenytoin and Bovine Serum Albumin
}

\author{
Mehrdad Hamidi', Kourosh Azimi ${ }^{1}$, Soliman Mohammadi-Samani ${ }^{2}$ \\ ${ }^{1}$ Department of Pharmaceutics, School of Pharmacy, Zanjan University of Medical Sciences, Zanjan, Iran; \\ 2 Department of Pharmaceutics, Faculty of Pharmacy, Shiraz University of Medical Sciences, Shiraz, Iran.
}

Received, November 11, 2010; Revised, January 29, 2011; Accepted, January 29, 2011; Published, January 30, 2011.

\begin{abstract}
The aim of the present study was to use a novel co-encapsulation method to prepare phenytoinloaded human erythrocytes with improved loading parameters and release profiles. Carrier erythrocytes coencapsulated by phenytoin and the model protein, bovine serum albumin, were prepared using the hypotonic pre-swelling method. A series of in vitro characterization tests were carried out on the carrier cells including loading parameters, drug and hemoglobin release, hematological indices, particle size analysis, osmotic fragility, turbulence fragility and scanning electron microscopy (SEM). Co-encapsulation with bovine serum albumin (BSA) resulted in about 8-times higher drug loading in erythrocytes with biphasic release trend instead of triphasic in the case of drug-alone loading. In comparison to the normal unloaded cells, hemoglobin content of the cells was decreased in the case of both drug and drug/protein loading, apparent cell sizes remained unchanged, cell shapes were changed to more spherical rather than biconcave discoid, and the osmotic as well as turbulence fragilities were higher in the case of drug/protein but were unchanged in the case of drug-alone loading. The most profound finding of this study was the possibility of achieving remarkably higher drug loading and more controllable drug release profile in the case of drug/protein loading with no unwanted in vitro characteristics change.
\end{abstract}

\section{INTRODUCTION}

Erythrocytes, as the most abundant and available cells in the human body have gained interest as cellular drug carriers in recent years (1-3). The main rationales for the use of this delivery system are to provide slow release of the loaded drugs in systemic circulation and/or to deliver drugs to specific sites within the body, mainly the organs of reticuloendothelial system (RES) (1). Biocompatibility, complete biodegradability, long life-span in circulation in comparison to the synthetic particulate carriers, protection of the organism against the toxic effects of the entrapped drug, and the possibility of targeted drug delivery to the RES organs are among the main advantages of this drug delivery system $(1,2)$. Two main drawbacks against the widespread use of the erythrocyte-based drug delivery systems for lipophilic drugs are their limited water solubility which prevents the achievement of a therapeutically reasonable drug loading, and the fast escaping of the loaded drug from the erythrocytes even during the early preparation stages $(4,5)$. The model drug used in this study, phenytoin, is one of the oldest but still widely used antiepileptic agents. The drug presents one of the main therapeutic challenges in antiepilepsy clinic because of its toxicity as well as unpredictable oral absorption and clearance $(6,7)$. By providing a near zero-order systemic input, the overall pharmacokinetic profile of the drugs is expected to become less variable. Thus, we selected phenytoin as a potential candidate for erythrocytebased intravenous (systemic) delivery. However, the successful use of erythrocytes for this purpose suffers from both the above-mentioned typical problems of lipophilic drugs as in early phases of this study we experienced that not only a therapeutically enough drug loading can not be achieved, but also the rate of drug release immediately after erythrocytes loading is too high to be used as a sustained drug reservoir. As a logical solution, reversible binding, i.e., complexation, of the drug with some complexing

Corresponding Author: Mehrdad Hamidi, Department of Pharmaceutics, School of Pharmacy, Zanjan University of Medical Sciences, Zanjan, Iran. E-mail: hamidim@zums.ac.ir 
agents co-encapsulated with the drug within the erythrocytes during the encapsulation procedure seems noteworthy as it can make a second release controlling level for the drug. In this study, we have exploited a novel co-encapsulation strategy via coencapsulation of bovine serum albumin (BSA) with phenytoin in human intact erythrocytes.

\section{MATERIALS AND METHODS}

\section{Chemicals}

Phenytoin sodium was kindly donated by Loghman Pharmaceuticals (Tehran, Iran). Bovine serum albumin was from Sigma (St. Louis, MO, USA; Art. No. A2153). Other chemicals and solvents were purchased locally.

\section{Preparation of human erythrocytes}

Blood samples were withdrawn by venipuncture from healthy male volunteers aged from 20 to 25 years using polypropylene disposable syringes connected to disposable $19 \mathrm{G}$ needles and transferred to pre-heparinized polypropylene test tubes. After centrifuging at $1000 \mathrm{~g}$ for $10 \mathrm{~min}$, the plasma portion and buffy coat were separated by aspiration. The remaining packed erythrocytes were washed three times using phosphate-buffered saline (PBS: NaCl, $150 \mathrm{mM}$; K2HPO4, $5 \mathrm{mM}$; pH 7.4). The standard blood sampling procedure used for taking erythrocytes samples from human volunteers was approved by Shiraz University of Medical Sciences Bioethics Committee.

\section{Preparation of phenytoin/BSA aqueous solution}

For the encapsulation purposes, an aqueous solution

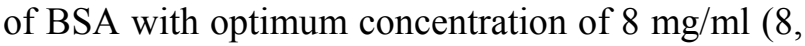
9) was prepared, in which different phenytoin concentrations (see the next sections) were dissolved. All BSA/drug solutions were prepared and used freshly.

\section{Co-encapsulation of phenytoin and BSA in human erythrocytes}

A hypotonic preswelling method described by Tajerzadeh and Hamidi (10) was used for loading the human erythrocytes with phenytoin or phenytoin/BSA throughout the experiments. For this purpose, $1 \mathrm{ml}$ of washed packed erythrocytes, prepared as described, was gently transferred to a polypropylene test tube, $4 \mathrm{ml}$ of a hypotonic PBS with an osmolarity of 0.67 -times of the eutonic solution was added, and the resulting cell suspension was mixed gently by 10-times tube inversions. The swollen cells obtained, were, then, separated by centrifuging at $1000 \mathrm{~g}$ for $10 \mathrm{~min}$ and the supernatant was discarded. A $200 \mu 1$ aliquot of a hemolysate, prepared by diluting a separate portion of the packed erythrocytes with distilled water (1:1) was gently added onto the remaining swollen cells. This lysate layer serves as the osmotic shock barrier as well as a reservoir for supplying the cell constituents being lost during the following drug addition steps. Then, $250 \mu \mathrm{l}$ of an aqueous solution of phenytoin or phenytoin/BSA aqueous solution was added gently onto the cell suspension, and the resulting mixture was inverted gently at least 10 times and centrifuged at $1000 \mathrm{~g}$ for $5 \mathrm{~min}$. Addition of drug solution, mixing, and centrifuging steps were repeated three more times to reach the lysis point of the cells. This point was detectable by a sudden increase in transparency of the cell suspension and the disappearance of a distinct boundary between cells and supernatant upon centrifuging. At this point, the erythrocytes were resealed by rapid addition of $100 \mu \mathrm{L}$ of hypertonic PBS with an osmolarity of 10-times of the eutonic solution, followed by gentle mixing of the suspension by several inversions. Finally, the resulting mixture was incubated at $37^{\circ} \mathrm{C}$ for $30 \mathrm{~min}$ to reanneal the resealed cells. The carrier erythrocytes obtained by this manner were washed three times using $10 \mathrm{ml}$ PBS to rinse out the unentrapped drug and the released hemoglobin and other cell constituents during the loading process.

When needed, sham-ecapsulated erythrocytes were prepared as described but with the protein/drug or drug aqueous solution replaced by distilled water.

\section{Loading parameters}

To evaluate the loading efficiency of the erythrocytes by phenytoin throughout the study, three parameters were defined and determined as loading parameters:

a. Loaded amount, the total amount of phenytoin encapsulated in the final packed erythrocytes;

b. Efficiency of entrapment, the percentage ratio of the loaded amount of phenytoin to the total amount added during the entire loading process;

c. Cell recovery, the percentage ratio of the hematocrit value of the final loaded cells to that of 
the initial packed cells, measured on equal suspension volumes.

\section{Effect of drug concentrations on loading parameters}

To evaluate the effect of phenytoin initial concentration on the loading parameters of the drug in erythrocytes, both in absence and in presence of BSA, a series of drug concentrations, including 10 , 25,50 and $100 \mathrm{mcg} / \mathrm{ml}$ in 'drug-alone' case and 100 , 250,500 and $750 \mathrm{mcg} / \mathrm{ml}$ in phenytoin/BSA case, were used during the loading procedure and the loading parameters were determined in each case.

\section{Mechanism of entrapment}

To investigate the possible mechanism of entrapment, an encapsulation procedure was performed, as described, and the drug concentrations in each of three final washing solutions as well as in final packed cells were determined. Then, the total amount of washed out (unentrapped) drug was calculated by considering the total volume of discarded solutions. On the other hand, the total amount of entrapped drug was determined using the loaded amount multiplied by the cell recovery of the method. Finally, taking the volume fraction of cells in whole suspension at the lysis point, the mechanistic behavior of drug entrapment in erythrocytes was evaluated.

\section{Drug Assay}

An isocratic reversed-phase HPLC method consisted of a mixture of phosphate buffer $\left(\mathrm{K}_{2} \mathrm{HPO}_{4}\right.$ $0.05 \mathrm{M} ; \mathrm{pH}=6.5)$ and methanol $(55: 45)$ as the mobile phase and a $\mathrm{C}_{18}$ analytical column (Tracer Excel; $150 \times 4.6 \mathrm{~mm}$; particle size $5 \mu \mathrm{m}$; Teknokroma, Spain) were used for determination of phenytoin concentrations in experimental samples. The column was operated at ambient temperature while connected by a guard column with the same packing (Teknokroma, Spain). The solvent delivery system used was a double-reciprocating pump (Knauer, model 100, Berlin, Germany). An ultraviolet detector (Knauer, model 2500, Berlin, Germany) operated at a wavelength of $210 \mathrm{~nm}$ was used for analyte detection with the outputs processed and recorded by a PC-compatible software (Knauer, Chromgate, Berlin, Germany). Sample injection was made by a loop injector (Rheodyne ${ }^{\circledR}$, Cotati, CA, USA) equipped by a 50 $\mu l$ loop. The method was linear throughout the wide phenytoin concentration range of 50-5000 ng/ml with the within-run and between-run variations being all less than 10 percent with the corresponding accuracies of more than 90 percent in all the cases. The drug assay in aqueous bufferic supernatants was performed by direct injection to chromatograph, while the packed cells were lysed prior to analysis, via dilution with an equal volume of distilled water and the lysate was deproteinized via vortex-mixing with an equal volume of acetonitrile.

\section{Characterization tests, in vitro}

\section{Protein and hemoglobin release}

To exploit the release kinetics of phenytoin as well as hemoglobin (an indicator of cell lysis kinetic) from carrier erythrocytes, 0.5 -ml portions of packed phenytoin- or phenytoin/BSA-loaded cells were diluted to $10 \mathrm{ml}$ using ringer solution containing $0.01 \%$ sodium azide $(\mathrm{NaN} 3)$, as an antimicrobial preservative, and the suspensions were mixed thoroughly by several gentle inversions. The resulting mixtures were divided into ten 1-ml portions in $1.5 \mathrm{~mL}$ polypropylene microtubes. The samples were, then, rotated vertically $(15 \mathrm{rpm})$ while incubated at $37^{\circ} \mathrm{C}$. At the beginning time of the test and also when $0.5,1,2,4,8,24,48,72 \mathrm{~h}$ and 7 days elapsed, one of the aliquots was harvested and after centrifuging at $1000 \mathrm{~g}$ for $5 \mathrm{~min}$, $100 \mu$ of the supernatants were used for phenytoin assay, as described earlier. In addition, the absorbance of another $0.5 \mathrm{ml}$ portion of the supernatants was determined in $540 \mathrm{~nm}$ using a UV/visible spectrophotometer (Cecil, model 9000, UK) to monitor the hemoglobin release. These experiments were carried out in triplicate and the percent of phenytoin and hemoglobin release was determined in reference to a completely lysed sample (100\% release) which was prepared by adding distilled water instead of ringer solution with the same volume ratio to a $0.1-\mathrm{ml}$ portion of the packed erythrocytes.

In another set of experiments, the same procedure was carried out as described except for the release media replaced by the autologous plasma.

\section{Hematological indices}

The hematological indices of four types of erythrocytes, i.e., phenytoin-loaded, phenytoin/BSA-loaded, sham-encapsulated, and 
unloaded, obtained from the same volunteer, were determined using a coulter counter-based instrument (Norahan Farjad, model MS9, Iran). The tested parameters consisted of mean corpuscular volume (MCV), mean corpuscular hemoglobin $(\mathrm{MCH})$ and mean corpuscular hemoglobin content (MCHC).

\section{Laser-assisted particle size analysis}

To investigate the effect of loading process on the particle size distribution of the erythrocytes population, a laser-based particle size analyzer (Shimadzu, model SALD- 2101, Japan) was used. For this purpose, phenytoin-loaded, phenytoin/BSA-loaded, sham-encapsulated, and unloaded cells were analyzed while suspended in normal saline in a dilution according to instrument operation conditions.

\section{Osmotic fragility}

To evaluate the resistance of erythrocytes membranes against the osmotic pressure changes of their surrounding media, $0.1-\mathrm{ml}$ aliquots of the packed erythrocytes of each type, i.e., phenytoinloaded, phenytoin/BSA-loaded, sham-encapsulated, and unloaded, were suspended in $1.5 \mathrm{ml}$ of $\mathrm{NaCl}$ aqueous solutions having different osmolarities between 0 to $300 \mathrm{mosm} / \mathrm{lit}$. After gentle $(15 \mathrm{rpm})$ vertical shaking at $37^{\circ} \mathrm{C}$ for $15 \mathrm{~min}$, the suspensions were centrifuged at $1000 \mathrm{~g}$ for $5 \mathrm{~min}$, and the absorbance of the supernatants determined spectrophotometrically at $540 \mathrm{~nm}$. The released hemoglobin was expressed as percentage absorbance of each sample in reference to a completely lysed sample prepared by diluting 0.1 $\mathrm{ml}$ of packed cells of each type with $1.5 \mathrm{ml}$ of distilled water instead of $\mathrm{NaCl}$ solutions. For comparative purposes, an osmotic fragility index (OFI) was defined in each case as the $\mathrm{NaCl}$ concentration producing $50 \%$ hemoglobin release (69).

\section{Turbulence fragility}

To exploit the mechanical strength of the erythrocytes membranes, $0.5-\mathrm{ml}$ samples of packed erythrocytes of each four types, were suspended in $10 \mathrm{ml}$ of PBS and were shaken vigorously using a multiple test tube orbital shaker (IKA, model VXR, Germany) at $2000 \mathrm{rpm}$ for $4 \mathrm{~h}$. To determine the timecourse of hemoglobin release, $0.5-\mathrm{ml}$ portions from each of the suspensions were withdrawn in 0 , $0.5,1,2$, and $4 \mathrm{~h}$, and after centrifuging at $1000 \mathrm{~g}$ for $5 \mathrm{~min}$, the absorbance of the supernatants were determined spectrophotometrically at $540 \mathrm{~nm}$. The percentage release of hemoglobin was determined in reference to a completely lysed cell suspension with the same cell fraction (i.e., $0.5 \mathrm{ml}$ of packed cells added to $10 \mathrm{ml}$ of distilled water). To compare the turbulence fragilities of the different types of erythrocytes, a turbulence fragility index (TFI) was defined as the shaking time producing $20 \%$ release of hemoglobin from the erythrocytes (69).

\section{Scanning Electron Microscopy (SEM)}

To investigate the possible morphological changes of erythrocytes upon loading process, samples of four types of erythrocytes were prepared and analyzed using an electron microscope (SEM 360, Cambridge, U.K.) after coating with gold particles by a Sputter Coater (Fisons, model 7640, U.K.) in $18 \mathrm{~mA}$ for 40 seconds.

\section{DATA ANALYSIS AND STATISTICS}

All the numerical results are presented as mean \pm SD. Statistical comparisons were performed using the two-tailed Student's t-test or analysis of variance (ANOVA) for more than two means followed by Tukey range test as the post-hoc test, using SPSS 12 for windows. The level of significance was set at 0.05 .

\section{RESULTS}

As indicated in Table 1, the loaded amount of phenytoin in human erythrocytes was significantly and substantially increased in the presence of BSA while the other two parameters, i.e., entrapment efficiency and cell recovery remained highly unchanged (Table 1). The loaded amount of the drug was increased significantly with the increasing initially used drug concentration (Table 2). From Table 3, the drug is highly recoverable quantitatively after the loading procedure. 
Table 1: Loading parameters of phenytoin in human intact erythrocytes using the hypotonic pre-swelling method in presence and in absence of BSA. Phenytoin concentration was $100 \mu \mathrm{g} / \mathrm{ml}$ and BSA concentration was $8 \mathrm{mg} / \mathrm{ml}$ in both cases $(\mathrm{n}=3)$

\begin{tabular}{lcc}
\hline Parameter & in absence of BSA & in presence of BSA $^{\mathbf{1}}$ \\
\hline Loaded Amount, $\mu \mathrm{g}$ & $8.77(0.64)$ & $67.68(1.38)^{*}$ \\
Entrapment Efficiency, \% & $8.77(1.14)$ & $8.98(0.94)$ \\
Cell Recovery (\%) & $71.91(1.68)$ & $72.35(1.29)$ \\
\hline *significantly different from values obtained in the absence of BSA; values in mean (SD). \\
\hline
\end{tabular}

\begin{tabular}{|c|c|c|c|c|}
\hline \multicolumn{5}{|l|}{ In absence of BSA: } \\
\hline Phenytoin, $\mu \mathrm{g} / \mathrm{ml}$ & 10 & 50 & 75 & 100 \\
\hline Loaded amount $(\mu \mathrm{g})$ & $1.98(0.13)^{*}$ & $4.09(0.41)^{*}$ & $6.67(1.23)^{*}$ & $8.77(0.64)^{*}$ \\
\hline Entrapment Efficiency (\%) & $19.8(0.09)^{*}$ & $8.18(0.57)$ & $8.89(1.11)$ & $8.77(1.14)$ \\
\hline Cell Recovery (\%) & $75.14(2.33)$ & $71.71(1.98)$ & $65.55(2.86)$ & $71.91(1.68)$ \\
\hline \multicolumn{5}{|l|}{ In presence of BSA $(8 \mu \mathrm{g})$ : } \\
\hline Phenytoin, $\mu \mathrm{g} / \mathrm{ml}$ & 100 & 250 & 500 & 750 \\
\hline Loaded amount $(\mu \mathrm{g})$ & $5.39(0.39)^{*}$ & $10.54(1.04) *$ & $36.53(1.32)^{*}$ & $67.68(1.38)^{*}$ \\
\hline Entrapment Efficiency (\%) & $5.39(0.36)^{*}$ & $4.20(0.43)^{*}$ & $7.24(0.33)^{*}$ & $8.77(0.64)^{*}$ \\
\hline Cell Recovery (\%) & $69.34(0.75)$ & $67.98(0.87)$ & $70.11(1.98)$ & $72.35(1.29)$ \\
\hline
\end{tabular}

\begin{tabular}{|c|c|c|c|}
\hline \multirow[t]{2}{*}{ Fraction } & \multirow{2}{*}{ Volume, $\mathrm{ml}$} & \multicolumn{2}{|c|}{ Phenytoin recovered } \\
\hline & & $\mu \mathrm{g} / \mathrm{ml}$ & $\mu \mathrm{g}$ \\
\hline $\begin{array}{l}\text { Total (pooled) washing } \\
\text { solutions }\end{array}$ & 30 & $22.6(2.51)$ & $676.8(24.4)$ \\
\hline $\begin{array}{l}\text { Final packed cell } \\
\text { Total amount recovered }\end{array}$ & $0.711(0.0125)$ & $86.4(1.34)$ & $\begin{array}{l}61.67(2.61) \\
730(31.6)\end{array}$ \\
\hline
\end{tabular}

\section{Drug and Hemoglobin Release}

The release profiles of phenytoin and hemoglobin from erythrocytes carrying phenytoin or $\mathrm{BSA} / \mathrm{Phenytoin}$, at $37^{\circ} \mathrm{C}$ in PBS are shown in Fig. 1. The corresponding release profiles in the autologous plasma are shown in Fig. 2. As shown, there are observable differences in release trends of the drug from drug-loaded and drug/protein-loaded erythrocytes.

\section{Hematological indices}

The hematological indices of four types of erythrocytes, i.e., phenytoin-loaded,
phenytoin/BSA-loaded, sham-encapsulated, and unloaded, obtained from the same volunteer, are shown in Table 4 . As shown by these data, For both the drug-loaded and drug0protein-loaded erythrocytes, $\mathrm{MCH}$ and $\mathrm{MCHC}$ are decreased significantly.

\section{Laser-assisted particle size analysis}

The volume-based size distribution data of four types of erythrocytes are shown in Table 5. No significant change was detectable between the cell sizes based on these measurements. 

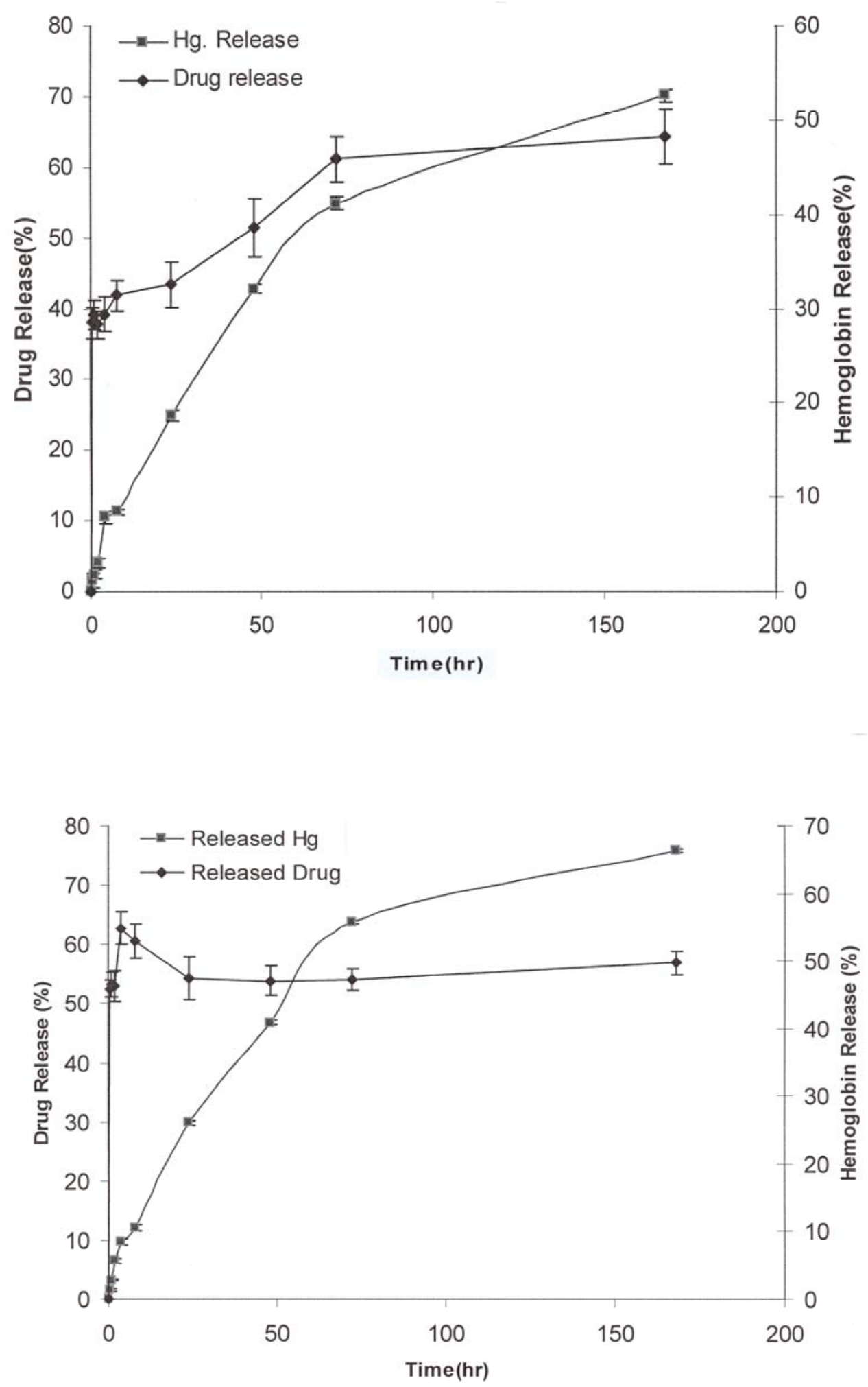

Figure 1. Release profiles of phenytoin and hemoglobin from carrier erythrocytes loaded by phenytoin (a) and BSA/phenytoin (b) in PBS bufferic medium ( $\mathrm{pH} 7.4)(\mathrm{n}=3)$. 

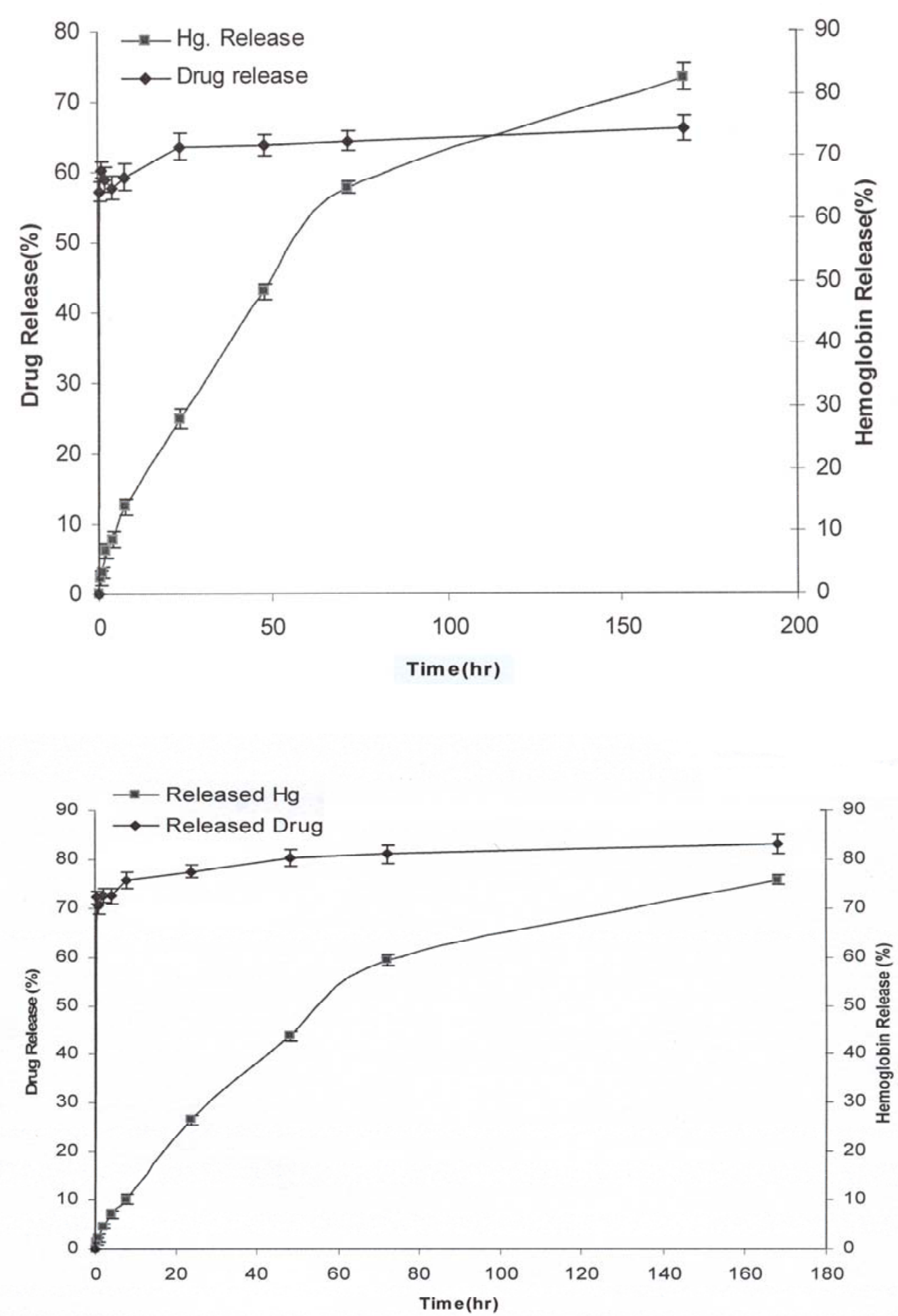

Figure 2. Release profiles of phenytoin and hemoglobin $(\mathrm{Hb})$ from carrier erythrocytes loaded by phenytoin (a) and BSA/phenytoin (b), in autologous plasma medium ( $n=3)$. 


\section{Scanning Electron Microscopy}

The SEM micrographs of four types of erythrocytes are shown in Fig. 3. The appearance of some cell shapes different from the expected normal biconcave, e.g., spherocytes and stomatocytes are evident from the micrographs.

\section{Osmotic fragility}

The osmotic fragility curves of four types of erythrocytes are shown in Fig. 4. Accordingly, the OFIs for unloaded, sham-encapsulated, phenytoinloaded and phenytoin/BSA loaded erythrocytes were determined as 153, 179, 158, and 190 mosm/lit, respectively. A change from the normal sigmoidal trend to a linear one is observable in the curves for the phenytoin/BSA-loaded and shamencapsulated cells.

\section{Turbulence fragility}

The turbulence fragility curves of four types of erythrocytes are shown in Fig. 5. Accordingly, the TFIs of unloaded, sham-encapsulated, phenytoinloaded and phenytoin/BSA loaded erythrocytes were determined as $3.67,2.38,3.26$, and $1.75 \mathrm{~h}$, respectively. As increased turbulence fragility of the cells is apparent from these data for the pheytoin/BSA-loaded group.

\begin{tabular}{lllll}
\hline \multicolumn{4}{l}{ Table 4. Hematological indices of four types of erythrocytes, determined by coulter-counter $(\mathrm{n}=3)}$. \\
\hline Hematological Index & $\begin{array}{l}\text { Unloaded } \\
\text { Erythrocytes }\end{array}$ & $\begin{array}{l}\text { Sham- } \\
\text { encapsulated } \\
\text { Erythrocytes }\end{array}$ & $\begin{array}{l}\text { Phenytoin-loaded } \\
\text { Erythrocytes }\end{array}$ & $\begin{array}{l}\text { Phenytoin/BSA- } \\
\text { loaded Erythrocytes }\end{array}$ \\
\hline MCV (fl) & $92.7(0.00)$ & $90.1(1.98)$ & $92.18(1.79)$ & $89.26(1.54)$ \\
MCH (pg) & $32.7(1.56)$ & $27.25(0.70) *$ & $26.34(0.31)^{*}$ & $25.98(0.67)^{*}$ \\
MCHC $^{\mathrm{f}}(\mathrm{g} / \mathrm{dl})$ & $35.3(1.70)$ & $30.22(0.17)^{*}$ & $26.40(0.91)^{*}$ & $25.29(0.98)^{*}$ \\
\hline
\end{tabular}

$\mathrm{MCV}$, mean corpuscular volume; fl, femtoliter; $\mathrm{MCH}$, mean corpuscular hemoglobin;pg, pictogram; MCHC, mean corpuscular hemoglobin content; g/dl, gram per deciliter. Values are mean (SD); * Significantly different from the unloaded cells.

\begin{tabular}{|c|c|c|c|c|c|}
\hline \multicolumn{2}{|c|}{ Statistical Parameters } & $\begin{array}{r}\text { Unloaded } \\
\text { Erythrocytes }\end{array}$ & $\begin{array}{r}\text { Sham-encapsulated } \\
\text { Erythrocytes }\end{array}$ & $\begin{array}{r}\text { Phenytoin-loaded } \\
\text { Erythrocytes }\end{array}$ & $\begin{array}{l}\text { BSA/phenytoin- } \\
\text { loaded Erythrocytes }\end{array}$ \\
\hline \multirow[t]{4}{*}{ Mean } & Mean $(\mu \mathrm{m})$ & $6.45(0.18)$ & $5.96(0.45)$ & $6.38(0.25)$ & $6.38(0.53)$ \\
\hline & SD & $0.076(0.002)$ & $0.075(0.006)$ & $0.076(0.002)$ & $0.078(0.002)$ \\
\hline & Median $(\mu \mathrm{m})$ & $6.55(0.03)$ & $6.18(0.53)$ & $6.44(0.26)$ & $6.47(0.24)$ \\
\hline & Mode $(\mu \mathrm{m})$ & $6.21(0.02)$ & $6.04(0.48)$ & $6.29(0.5)$ & $6.32(0.1)$ \\
\hline
\end{tabular}




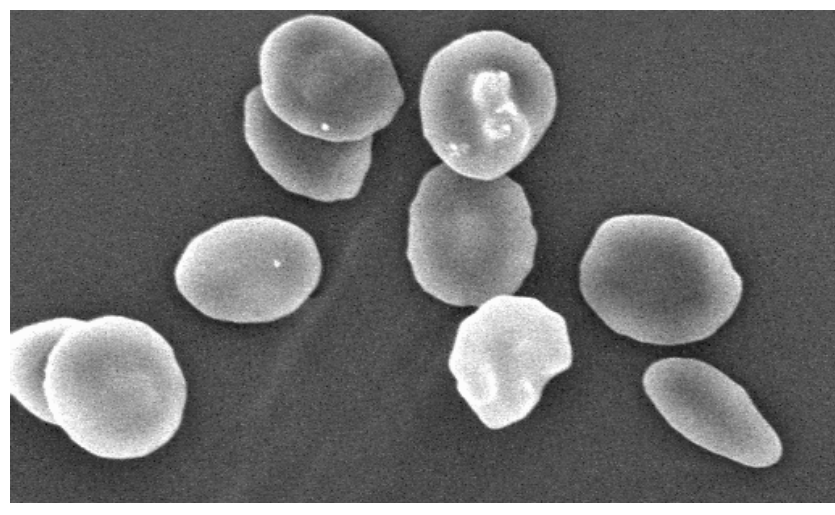

A

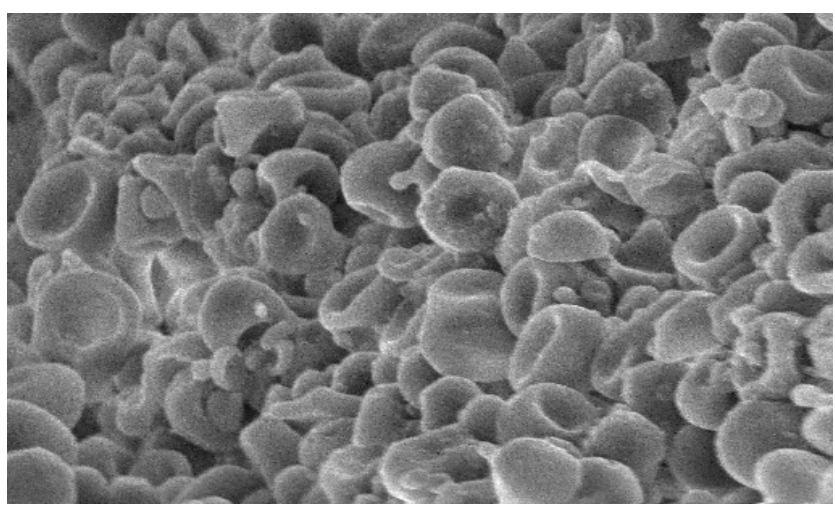

$\mathrm{C}$

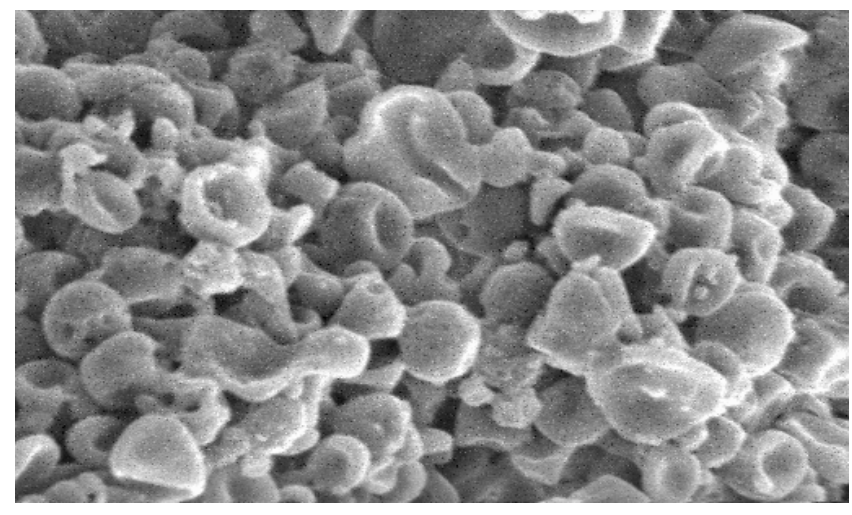

B

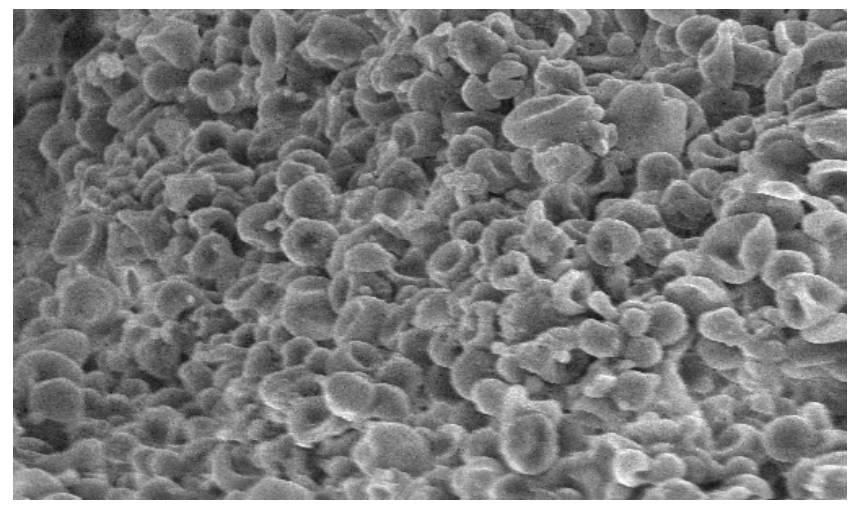

D

Figure 3. Scanning electron microscopy (SEM) images of a) unloaded $(\times 3500)$, b) sham-encapsulated $(\times 2000)$, c) phenytoin-loaded $(\times 2000)$, and d) phenytoin/BSA-loaded erythrocytes $(\times 2000)$. For panel A, $1 \mathrm{~cm}$ represents $2.86 \mu \mathrm{m}$, for the rest, $1 \mathrm{~cm}$ represents $5 \mu \mathrm{m}$.

\section{DISCUSSION}

Phenytoin is among the oldest, but still widely used antiepileptic drugs with well-documented nonlinear, i.e., saturable, pharmacokinetic behavior within the therapeutic concentration range. The rationale for developing an intravenous long-term controlled release delivery system for this drug is that if somehow we can control the rate of drug entry to circulation, the input function of the drug becomes near zero-order which, in turn, makes the disposition factor more predictable by maintenance of the drug systemic concentration in a narrow range thereby decreasing the possibility of drug clearance saturation. This strategy, which is based on the pharmacokinetic variations rather than formulation-dependent variations of the biofate of the drug, is the basis for the development of the majority of the cellular carriers. Phenytoin tends to readily escape rapidly from the loaded cells. Therefore, a model protein, BSA, was incorporated in the drug loaded cells in order to increase the loaded amount and also the drug retention time within erythrocytes. BSA is a well-characterized protein with high binding affinity and capacity for phenytoin (our preliminary studies) and a good potential for being encapsulated in human erythrocytes $(7,8)$. In fact, the rationale for this strategy was: i) to reach higher drug loading via achievement of higher initial drug concentrations used during the loading procedure, and ii) to keep the drug within the cells for longer time by providing an additional level of drug binding to the protein entrapped within the cells during the loading process. 


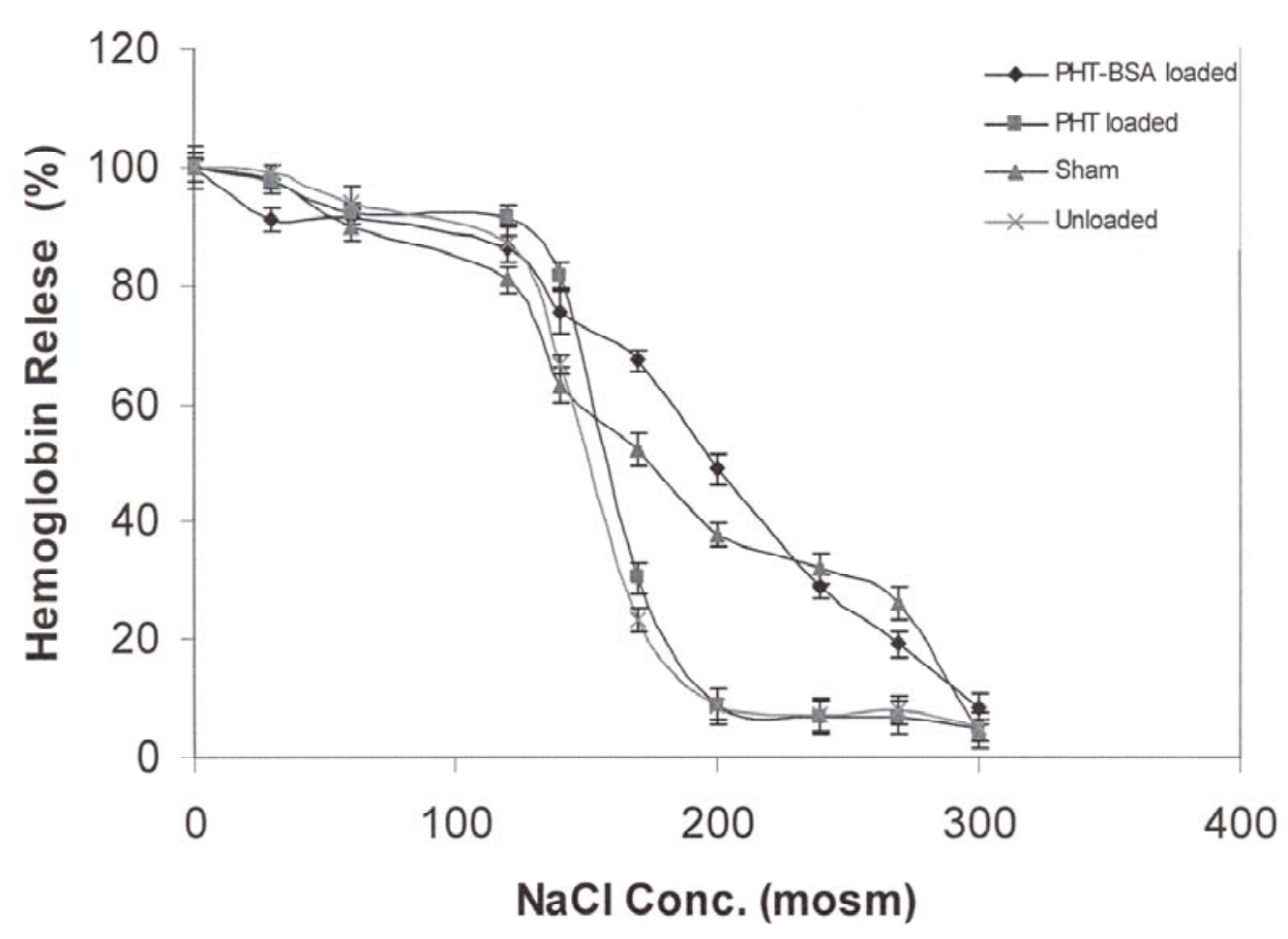

Figure 4. Osmotic fragility curves of unloaded, sham-entrapped, phenytoin-loaded and phenytoin-BSA loaded erythrocytes prepared by hypotonic preswelling method $(\mathrm{n}=3)$.

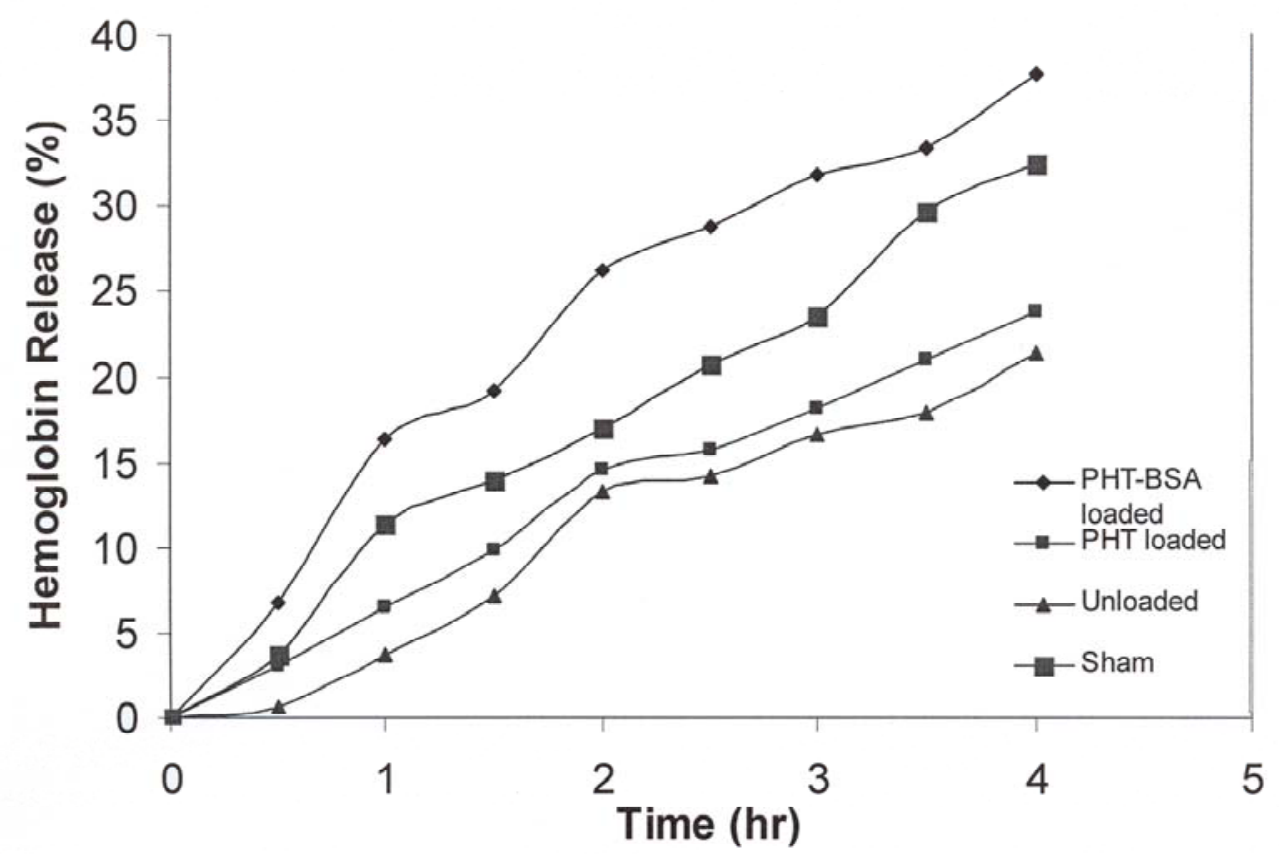

Figure 5. Turbulence fragility curves of unloaded, sham-entrapped, phenytoin-loaded and phenytoin-BSA loaded erythrocytes prepared by hypotonic preswelling method $(n=3)$. 
The BSA co-encapsulation increased pheytoin loaded amount in erythrocytes about 8-times (Table 1). This, in turn, ensures sufficient entry of the drug to the host body upon injection of relatively low volumes of loaded packed cells to the blood circulation. The encapsulation efficiencies of the drug in erythrocytes, however, were closely similar in presence and in absence of BSA, indicating that the only parameter involved in the achievement of higher drug loading in protein/drug case is the improvement of drug total solubility via complexation with protein. Furthermore, it can be concluded from these data that there is a highcapacity reversible 'binding site' in/on erythrocytes for phenytoin, which governs the final equilibrium state between bound (i.e., loaded) and unbound (i.e., unloaded) drug with the bound ratio of about 0.09 both with and without protein. A cell recovery of about $70 \%$ in both cases, i.e., with and without BSA, is comparable to recovery result for other drugs and proteins observed previously (e.g., 8, 1113) and, on the other hand, ensures that, as expected, the inclusion of BSA in loading procedure do not result in any observable harm to cells. The encapsulation efficiency of about 9 percent needs to be improved further and, in fact, is under investigation in our lab by different strategies, e.g., membrane crosslinking with glutharaldehyde.

The effect of phenytoin concentration on the loaded amount of the drug was linear in absence of BSA (Table 2). However, this trend shows a disproportional increasing trend in the presence of BSA (Table 2), with the loaded amount increased higher-than-expected, which can be explained by the involvement of some saturable competing binding sites for phenytoin in/on erythrocytes, leading to progressingly higher intracellular drug concentrations with increasing drug concentrations used in the loading procedure.

Considering the loaded and washed out (unloaded) amounts of the drug determined after completion of the loading procedure (Table 3 ), it is clear that the main mechanism for drug entrapment in erythrocytes is simple concentration gradientbased diffusion with drug partitioning between inside and outside of the cells governed only by the volume fraction of the cells in total cell suspension at the lysis point (see encapsulation procedure in Materials and Methods).
Hemoglobin release from both types of loaded erythrocytes, i.e., with and without BSA, shows a triphasic profile represented by a curve with three distinct slopes. The first segment indicates the most fragile cells breakdown and includes the first few hours of the release test period; erythrocytes with higher resistance against environmental conditions form the second part of the curve which extends up to about $50 \mathrm{~h}$; and, finally, the hemoglobin release occurs very slowly from cells undergone minimal structural changes during the loading procedure which is represented by a slow release phase up to the end of the test time. This trend was evident in both release media, i.e., plasma and PBS (Figs, 1 and 2).

The release profile of phenytoin from phenytoin-alone--loaded erythrocytes consists of three clearly distinguishable phases in PBS release medium. (Fig. 1). An initial apparent burst release of a considerable amount of the loaded drug was evident during the first few hours of the experiments. Since the drug release trend of this segment is not consistent with the hemoglobin release trend (i.e., the hemolysis curve), it can be attributed to a part of the drug weakly bound or unbound to the cell sub-structures, thus readily diffusible out of the intact cells. The second release phase can be probably_ascribed to a part of the drug bound to a high-affinity reversible binding site within the erythrocytes, with the drug release rate based on the equilibrium state between the free and bound drug. The third delayed phase of the drug release seen in PBS is relatively consistent with the final part of hemoglobin release profile and, thus, indicates the hemolysis-dependent drug release. This finding can be attributed probably to the presence of an irreversible binding site for phenytoin which prevents the drug release, in part, from the intact cells prior to cell destruction but the drug is allowed to come out after cell destruiction.

Co-encapsulation of BSA with drug has resulted in higher initial fast release in PBS (about 10 percent higher) along with the disappearance of the second "reversible binding phase" (Fig. 1). Based on this finding, it seems that BSA displaces the drug from its reversible cellular binding site, thereby making it available for both fast release and irreversible binding. Consequently, the drug shows an about 50 percent fast release with the rest of the loaded amount being released very slowly consistent to the cell lysis profile (Fig. 1). The same 
behavior from drug-loaded and drug/protein-loaded erythrocytes was evident in autologous plasma release medium; again, more initial fast release as well as disappearance of the second release phase was observed in presence of BSA (Fig. 2). Furthermore, with suspending the phenytoin-loaded or phenytoin/BSA-loaded erythrocytes in the plasma medium, a significantly higher amount of the drug was released compared point-to-point throughout the release experiment period. This observation can be clearly explained by the presence of high-affinity binding entities for phenytoin in plasma surrounding the cells, presumably plasma albumin, which shifts the drug partitioning equilibrium outward. In other words, these binding functions 'pull' the drug out of the erythrocytes into the surrounding medium.

Therefore, the net result of BSA coencapsulation with phenytoin in erythrocytes with respect to drug release behavior would be transformation of an apparent triphasic release profile to a biphasic one (Figs. 1 and 2) characterized by a higher initial fast drug release as well as a more predictable delayed-release phase which is governed, mainly, by the cell lysis trend in circulation rather than a hybrid phenomenon of reversible binding and cell lysis as in the case of drug-alone loading. It is obvious that the cell lysis is a more robust and predictable phenomenon than the reversible cell binding, which can be influenced by different factors.

In all release experiments, the profile (Figs. 1 and 2) approaches an apparent plateau after 24-48 hours, which may be explained by the establishment of a balance between drug slow release and the documented drug decomposition in experimental condition.

Based on the overall drug release data, while the overall idea of intracellular drug complexation seems to be beneficial in terms of modifying the drug release pattern, BSA seems not to be a suitable candidate for phenytoin co-encapsulation considering the net drug release profile. We believe that the main reason for this observation would be the weak affinity of the drug to BSA which, in turn, brings in mind the replacement of BSA with some 'stronger' drug-complexing agents to keep the drug inside the cells for longer time periods to achieve a slow release system in practice.

With respect to the hematological indices tested routinely in clinical laboratories, three parameters, mean corpuscular volume (MCV), mean hemoglobin content $(\mathrm{MCH})$, and mean corpuscular hemoglobin content (MCHC) were determined for four types of erythrocytes, i.e., unloaded, shamencapsulated, phenytoin-loaded, and phenytoin / BSA-loaded. While no significant changes were evident in MCV values upon loading procedure, both without drug or with the drug or with drug/BSA loading, the parameters indicating the cellular hemoglobin content, $\mathrm{MCH}$ and $\mathrm{MCHC}$, decreased significantly upon loading procedure in all three variants. In other words, while the mean cell sizes determined by this coulter-based technique did not changed upon loading, the hemoglobin loss during the loading procedure was significant, as expected from the partly hemolyzing nature of the loading procedure. In other studies $(14,15)$ all these parameters were decreased in carrier erythrocytes compared to the normal unloaded cells.

The laser diffraction-based cell size measurements on four cell types showed no significant differences between all cell types (Table 5), which, again, confirms that the average cell diameters did not change upon loading procedure. An average cell size of about $6 \mu \mathrm{m}$ was consistent to our knowledge of erythrocyte dimensions and morphology. None of the statistical parameters of the cell diameters showed significant changes upon loading procedure.

As seen in SEM micrographs (Fig 3), the hypotonic preswelling method used in this tudy for drug or drug/protein encapsulation resulted in dramatic morphological/topological changes in erythrocytes with a general shape change from biconcave discoids in the case of normal unloaded cells to rather spherical cells, referred to as spherocytes, with some stomatocytes, i.e., spherical cells with an invagination at a point. The same shape changes have been reported previously by our group $(9,11,16)$. The average sphere sizes bserved in this test was consistent to the findings of other sections of this study, i.e., laser-based or coulter-based size analysis. The other major finding from SEM results is that the changes in cell shapes is a result of loading procedure itself (Fig. 3, sham group) with no discernible changes resulted from the incorporation of the drug or drug/protein. SEM studies by other groups has revealed that the majority of these cells maintain their normal biconcave discoid shapes after exposure to the 
loading procedure (15) with some "spherocytes" (17) and a few "stomatocytes" and, in some cases, a few microcytic erythrocytes (erythrocytes with smaller sizes than the normal cells) (18).

The osmotic fragility indices of unloaded, sham-entrapped, phenytoin-loaded and phenytoinBSA-loaded erythrocytes were graphically determined as 153, 179, 158 and $190 \mathrm{mosm} / \mathrm{L}$, respectively (Fig. 4). From these data, it is clear that the loading procedure without the drug or protein, results in more fragile cells against the osmotic pressure changes $(\mathrm{P}<0.05)$, an expected finding considering the destructive nature of the loading method used. However, loading by the drug alone resulted in erythrocytes that react to the osmolarity changes of the surrounding media in such a manner similar to the normal cells. Incorporation of phenytoin-BSA in cells, again, increased the osmotic fragility of the cells to an extent even more than sham encapsulation (Fig 4). Some 'anchoring' effect via phenytoin binding function and some steric disturbing effects by BSA incorporation can be responsible, at least in part, for these stabilizing or destabilizing effects. As it has also been reported previously by our group for other drugs $(9,11,13)$, the trends of osmotic fragility curves, have changed from sigmoidal in the case of unloaded cells to linear in the case of both phenytoin-BSA-loaded and sham-ecapsulated erythrocytes. This is indicative of more heterogeneous cell population in terms of osmotic fragility in the cases of drug-BSAloaded and sham-operated cells, caused by the loding procedure. Interestingly, the osmotic fragility trend for phenytoin-loaded erythrocytes, shows sigmoidal curve, indicating some kind of specific stabilizing effect of the drug on the cell structure (Fig. 4). A reduction in the average cell volume upon loading procedure, leading to a decrease in the internal pressure cannot be ruled out. In most of the studies testing

this parameter, osmotic fragility of the carrier erythrocytes has been higher than the normal unloaded cells along with a change in the trend of fragility curves from sigmoidal to some linear curves $(17,19,20)$.

Turbulence fragility of the erythrocytes, being a direct measure of the cell membrane mechanical strength (1), showed significant increase in shamencapsulated as well as phenytoin/BSA -loaded erythrocytes (Fig. 5). However, the turbulence fragility curve of the phenytoin-loaded cells were, again, closely similar to that of the unloaded cells with no significant changes in TFI values. This observation confirms the data in osmotic fragility test in that there is a stabilizing function by phenytoin on erythrocyte membrane which is not evident in BSA/drug-loaded cells.

\section{CONCLUSION}

A novel approach, co-encapsulation of a drug and a protein in erythrocytes, has been exploited in this study with the aim of improving both the drug loading parameters and release characteristics of the loaded drug. Overall, the data presented in this study show that, firstly, the loaded amount of phenytoin in human erythrocytes increased remarkably, about 8-times, mostly because of the enhanced drug apparent aqueous solubility; secondly, inclusion of BSA in loading procedure changed the release behavior of phenytoin from a triphasic profile to a biphasic one via displacing the drug from a cellular binding site, thus making the drug available for two other release modes of fast release or cell destruction-dependent very slow release. The latter is beneficial in time-controlled predictable systemic drug release; thirdly some in vitro characterization tests have shown irreversible changes upon loading procedure both with and without drug loading, including shape and membrane fragility of loaded erythrocytes. The possible effects of these changes on the drug delivery efficiency of these cellular carriers remain to be evaluated by further studies.

\section{REFERENCES}

1. Hamidi M, Tajerzadeh H, (2003) Carrier erythrocytes: an overview, Drug Delivery, 10: 9-20.

2. Hamidi M, Zarrin A, Foroozesh M, MohammadiSamani S, (2007) Applications of carrier erythrocytes in delivery of biopharmaceuticals, $J$. Control. Rel., 118: 145-160.

3. Banker GS, Rhodes CT, (2002) Modern Pharmaceutics, $4^{\text {th }}$ edn. New York, Marcel Dekker, p. 560.

4. Eichler HC, Gasic S, Daum B, Bacher S, Steger G, (1987) In vitro drug release from human carrier erythrocytes, Adv. Biosci. (series), 67: 11-15.

5. Eichler HG, Raffesberg W, Gasic S, Korn A, Bauer K, (1985) Release of vitamine B12 from carrier erythrocytes in vitro, Res. Exp. Med., 185: 341-344.

6. http://www.rxlist.com 
7. Martindale: The complete drug reference, (2007) 35th edn., London, Pharmaceutical Press.

8. Hamidi M, Zarei N, Zarrin A, Mohammadi-Samani S, (2007) Preparation and validation of carrier human erythrocytes loaded by bovine serum albumin as a model antigen/protein, Drug Delivery, 14: 1-6.

9. Hamidi M, Zarei N, Zarrin A, Mohammadi-Samani $S$, (2007) Preparation and in vitro characterization of carrier erythrocytes for vaccine delivery, Int. J. Pharm., 338: 70-78.

10. Tajerzadeh H, Hamidi M, (2000) Evaluation of hypotonic preswelling method for encapsulation of enalaprilat in intact human erythrocytes, Drug Devel. Ind. Pharm., 26: 1247-1257.

11. Hamidi M, Zarrin A, Foroozesh M, Zarei N, Mohammadi-Samani S, (2007) Preparation and in vitro evaluation of carrier erythrocytes for REStargeted delivery of interferon-alpha $2 \mathrm{~b}$, Int. J. Pharm., 341: 125-133.

12. Talwar N, Jain NK, (1992) Erythrocytes as carrier of primaquin preparation: characterization and evaluation, J. Control. Rel., 20: 133-142.

13. Magnani, M., Rossi, L., Fraternale, A., Bianchi, M., Antonelli, A., Crinelli, R., and Chiarantini, L. (2002). Erythrocyte-mediated delivery of drugs, peptides and modified oligonucleotides. Gene Ther. 9:749-751.
14. Baker, R.F., (1967) Entry of ferritin into red cells during hypotonic hemolysis. Nature 215: 424-425.

15. Deloach, J.R., Doleskey, R., (1987). Preparation and properties of microcytic carrier erythrocytes from sheep and goats. Adv. Biosci. (Ser.) 67, 199-212.

16. Hamidi M, Tajerzadeh, Dehpour AR, Rouini MR, Ejtemaee-Mehr Sh, (2001) In vitro characterization of human intact erythrocytes loaded by enalaprilat, Drug Delivery, 8: 223-230.

17. Garin, M.I., Lopez, R.M., Sanz, S., Pinilla, M., Luque, J., (1996) Erythrocytes as carriers for recombinant human erythropoietin. Pharm. Res. 13: 869-874.

18. Kruse, C.A., James, G.T., Freehauf, C.L., Williams, C.M., (1987). Methotrexate loaded erythrocytes carriers: optimization their formation, their characterization, and their pharmacological efficiency in treating hepatoma 129 ascites tumors in mice. Adv. Biosci. (Ser.) 67: 137-144.

19. Kinosita, K., Tsong, T.Y., (1977). Formation and resealing of pores of controlled sizes in human erythrocyte membrane. Nature 268, 438-440.

20. Jain, S., Jain, S.K., Dixit, V.K., 1997. Magnetically guided rat erythrocytes bearing isoniazid: preparation, characterization, and evaluation. Drug Devel. Ind. Pharm. 23, 999-1006. 